\title{
Efeito dos ácidos ascórbico e cítrico em propriedades físico-químicas e sensoriais de bolos vegetarianos com suplementação de farinha de bagaço de uva
}

\author{
Effect of ascorbic and citric acids on physicochemical and \\ sensory properties of vegetarian cakes supplemented with \\ grape marc flour
}

\author{
Nataly Roberta Bezerra Santana Carlini ${ }^{1,2 *}$ (D), Victória Zagna dos Santos ${ }^{3}$, \\ Claudileide de Sá Silva ${ }^{4}$, Márlon de Castro Vasconcelos ${ }^{5}$, Adriano Brandelli ${ }^{6}$, \\ Voltaire Sant'Anna ${ }^{7}$ (i)
}

\author{
${ }^{1}$ Universidade Estadual do Rio Grande do Sul (UERGS), Programa de Pós-graduação em Ambiente e \\ Sustentabilidade (Mestrado Profissional), São Francisco de Paula/RS - Brasil \\ ${ }^{2}$ Instituto Federal de Educação, Ciência e Tecnologia do Sertão Pernambucano, Petrolina/PE - Brasil \\ ${ }^{3}$ Universidade Estadual do Rio Grande do Sul (UERGS), Laboratório de Processamento de Resíduos de Alimentos, \\ Encantado/RS - Brasil \\ ${ }^{4}$ Universidade Estadual de Pernambuco (UPE), Colegiado de Nutrição, Petrolina/PE - Brasil \\ ${ }^{5}$ Universidade Estadual do Rio Grande do Sul (UERGS), Três Passos/RS - Brasil \\ ${ }^{6}$ Universidade Federal do Rio Grande do Sul (UFRGS), Instituto de Ciência e Tecnologia de Alimentos, Laboratório \\ de Bioquímica e Microbiologia Aplicada, Porto Alegre/RS - Brasil \\ ${ }^{7}$ Universidade Estadual do Rio Grande do Sul (UERGS), Programa de Pós-graduação em Ambiente e \\ Sustentabilidade (Mestrado Profissional), São Francisco de Paula/RS - Brasil e Laboratório de Processamento de \\ Resíduos de Alimentos, Encantado/RS - Brasil
}

*Corresponding Author: Nataly Roberta Bezerra Santana Carlini, Universidade Estadual do Rio Grande do Sul (UERGS), Unidade Encantado, Rua Alegrete, 821, CEP: 95960-000, Encantado/RS - Brasil, e-mail:

natalyrbs@gmail.com

Cite as: Carlini, N. R. B. S., Santos, V. Z., Silva, C. S., Vasconcelos, M. C., Brandelli, A., \& Sant'Anna, V. (2021).

Effect of ascorbic and citric acids on physicochemical and sensory properties of vegetarian cakes supplemented with grape marc flour. Brazilian Journal of Food Technology, 24, e2020243. https://doi.org/10.1590/1981-

6723.24320

\begin{abstract}
Resumo
O objetivo deste trabalho foi desenvolver formulações de misturas para preparo de bolos de caneca, sem ovos e sem leite, com farinhas de cascas e sementes de uvas, além de avaliar características físico-químicas, tecnológicas e sensoriais, com enfoque nos efeitos dos ácidos ascórbico e cítrico sobre os bolos, com substituição parcial da farinha de trigo por farinha de bagaço de uva. Aos produtos, foram incorporados $5 \%$ de farinhas de cascas e sementes em relação à farinha de trigo. Foram realizadas análises de polifenóis, atividade antioxidante, cor, $\mathrm{pH}$, volume específico, perda de massa e sensorial. Entre as formulações com farinha de uva, a amostra com adição de ácido ascórbico apresentou maior $(p<0,05)$ atividade antioxidante, medida pelo método ABTS, do que as amostras sem adição de ácidos. Entretanto, a formulação não apresentou diferença significativa $(p>0,05)$ quando comparada com a formulação com a adição de ácido cítrico ou a
\end{abstract}


combinação de ácidos. De acordo com o coeficiente de correlação de Pearson, quanto mais ácido (menor $\mathrm{pH}$ ) se mostrou o bolo, maior foi a tendência de aumento na concentração de polifenóis totais, antocianinas e compostos com atividade antioxidante. Com relação ao volume específico, a maioria das formulações controles apresentou os maiores volumes em relação às suas respectivas amostras com farinha de uva. Assim, a incorporação dos ácidos ascórbico e cítrico nos bolos com farinha de bagaço de uva possivelmente acarretou estabilização da coloração vermelha e o bolo com farinha de bagaço de uva e ácido ascórbico apresentou as maiores quantidades de compostos fenólicos totais, quando comparado aos outros bolos com o subproduto, além de ter sido um dos bolos mais bem aceitos pelos provadores, demonstrando o potencial de mercado para esses produtos alimentícios inovadores.

Palavras-chave: Bolo; Ecoinovação; Fenólicos; Indústria; Mercado; Subproduto; Sustentabilidade.

\begin{abstract}
This study aimed to develop formulations of mixtures to prepare mug cakes, without eggs and milk, with grape skin and seed flours, and to evaluate their physicochemical, technological and sensory characteristics focusing on the effects of ascorbic and citric acids on cakes with partial replacement of wheat flour with grape marc flour. The products were incorporated with $5 \%$ grape marc flour in relation to wheat flour. The following analyses were performed: polyphenols, antioxidant activity, color, $\mathrm{pH}$, specific volume, loss of mass, and sensory. Among the formulations with grape marc flour, the sample with addition of ascorbic acid showed higher $(p<0.05)$ antioxidant activity measured by the ABTS method than the samples without addition of acids. However, this formulation showed no statistically significant difference $(p>0.05)$ compared with those with addition of citric acid or a combination of acids. According to the Pearson's correlation coefficient, the more acidic (lower $\mathrm{pH}$ ) the cake was, the greater the tendency for increased concentration of total polyphenols, anthocyanins, and compounds with antioxidant activity. Regarding specific volume, most of the control formulations presented higher values compared with those of their respective samples with grape marc flour. Thus, incorporation of ascorbic and citric acids into cakes with grape marc flour possibly resulted in red color stabilization, and the cake with grape marc flour and ascorbic acid presented the highest amounts of total phenolic compounds compared with those of other cakes with this by-product, in addition to being one of the cakes most accepted by the tasters, demonstrating the market potential of these innovative food products.
\end{abstract}

Keywords: Cake; Eco-innovation; Phenolics; Industry; Market; By-product; Sustainability.

\title{
1 Introdução
}

A gestão agroindustrial de resíduos é um dos maiores desafios das indústrias de alimentos e, no caso da produção vinícola, o bagaço de uva representa cerca de 30\% da massa de uvas utilizadas, sendo uma ótima fonte de compostos bioativos. Ele retém grande quantidade de compostos fenólicos e estima-se que em torno de $20 \%$ a $30 \%$ estejam nas cascas, e $60 \%$ a $70 \%$ nas sementes (Monrad et al., 2010). Fatores, como a variedade da uva, o solo, o clima, a safra e o tipo de processamento utilizado, influenciam a composição do subproduto gerado. Por exemplo: no processamento do vinho branco, há geração de bagaço não fermentado, o qual possui composição química diferente do subproduto fermentado, proveniente do processo de vinificação tinta (Tonon et al., 2018). Isto ocorre porque quanto maior o tempo de contato do mosto com o bagaço, maior é a extração dos componentes para a bebida (Ishimoto, 2008).

$\mathrm{O}$ consumo de alimentos ricos em polifenóis tem sido associado à redução de riscos no desenvolvimento de doenças neurológicas, doença cardíaca, aterosclerose, câncer e diabetes (Toaldo et al., 2015). Outro aspecto importante deste resíduo é sua alta concentração de fibras, representando cerca de $60 \%$ de sua massa seca (Valiente et al., 1995). Observa-se que, após a vinificação, as fibras possuem características estruturais diferentes das fibras alimentares comuns, 
estando associadas a compostos fenólicos, sendo denominadas de fibras alimentares antioxidantes (Saura-Calixto, 1998; Sant'Anna et al., 2012). Com isso, tem sido valorizado o perfil nutricional do bagaço de uva, já podendo ser encontrados produtos à base deste subproduto da indústria vinícola em mercados ao redor do mundo (Monagas et al., 2006).

Aliado a este contexto, a demanda dos consumidores por panificados com maior teor de compostos bioativos, fabricados a partir de farinhas ricas em fibras e polifenóis, é crescente, desafiando a área de alimentos no desenvolvimento de novos produtos com essas características (Žilić et al., 2016). A adição de farinha de bagaço de uva em produtos de panificação pode ser uma alternativa para suprir esse mercado.

Uma possibilidade que tem mostrado viabilidade tecnológica e econômica é o enriquecimento nutricional de produtos de alta aceitação e consumo pela população com farinhas mistas elaboradas com resíduos industriais de frutas e hortaliças (Santana et al., 2011). Neste contexto, alguns trabalhos têm evidenciado alternativas para o aproveitamento do subproduto da uva e estudos têm avaliado sua aplicação em diversos alimentos, como muffins (Ortega-Heras et al., 2019), doce tipo brigadeiro (Cabral et al., 2020), entre outros. Apesar da grande diversidade de alternativas abordadas, ainda não foi verificado ensaio para sua utilização em mistura para preparo de bolo de caneca, sem ingredientes de origem animal. Com vistas à grande tendência na área de alimentos por produtos convenientes, práticos e saudáveis (Révillion et al., 2020), a incorporação de farinha de bagaço de uva em panificáveis, além de aumentar suas alternativas de destinação, contribuindo para o desenvolvimento de produtos funcionais, possibilita seu consumo por pessoas vegetarianas, uma vez que, segundo Révillion et al. (2020), o mercado para esse grupo de consumidores é crescente.

No entanto, é necessário haver estudos sobre a substituição parcial da farinha de trigo por farinha de bagaço de uva e seus impactos tecnológicos e sensoriais. O uso de aditivos em produtos de panificação pode auxiliar as indústrias de alimentos na melhoria das características reológicas da farinha de trigo em produtos que tiveram parte dessa matéria-prima substituída, sendo o ácido ascórbico o agente oxidante mais comumente usado para atuar sobre a estrutura das proteínas do glúten, conferindo fortalecimento à massa (Pereira et al., 2009).

Assim, este estudo tem por objetivo desenvolver formulações de misturas para preparo de bolos de caneca, sem ovos e sem leite, com adição de farinhas de cascas e sementes de uvas, e avaliar características físico-químicas, tecnológicas e sensoriais, com enfoque nos efeitos dos ácidos ascórbico e cítrico sobre os bolos, com substituição parcial da farinha de trigo por farinha de bagaço de uva.

\section{Materiais e métodos}

\subsection{Desenvolvimento dos bolos de caneca}

Para a elaboração dos produtos, foram utilizados protocolos pré-estabelecidos com a incorporação de $5 \%$ de farinhas de cascas e sementes (1:1) de uvas Bordô (Vitis labrusca L.) (Uva Só, EcoNatura, Garibaldi-RS) em relação à farinha de trigo. Os bolos foram preparados a partir da união dos seguintes ingredientes: farinha de trigo, açúcar demerara, fermento químico em pó (amido de milho geneticamente modificado, bicarbonato de sódio, fosfato monocálcico e carbonato de cálcio - Mondeléz, Curitiba, Brasil), farinhas de cascas e sementes de uvas (provenientes da produção de uvas orgânicas), ácido cítrico (Adicel, Belo Horizonte, Brasil) ou ácido ascórbico (Adicel, Belo Horizonte, Brasil) ou os dois, água potável e óleo de soja, como exposto na Tabela 1. No entanto, para a proposta comercial do produto, os dois últimos ingredientes devem ser adicionados pelos próprios consumidores. 
Efeito dos ácidos ascórbico e cítrico em propriedades físico-químicas e sensoriais de bolos vegetarianos com suplementação de farinha de bagaço de uva

Carlini, N. R. B. S. et al.

Tabela 1. Composição dos bolos com substituição parcial de farinha de trigo por farinha de bagaço de uvas, com ou sem aditivos, e seus respectivos controles.

\begin{tabular}{ccccccccc}
\hline Ingredientes & $\mathbf{A 1}$ & $\mathbf{C 1}$ & $\mathbf{A 2}$ & $\mathbf{C 2}$ & $\mathbf{A 3}$ & $\mathbf{C 3}$ & $\mathbf{A 4}$ & $\mathbf{C 4}$ \\
\hline Farinha de trigo & $41 \mathrm{~g}$ & $43 \mathrm{~g}$ & $40 \mathrm{~g}$ & $42 \mathrm{~g}$ & $38 \mathrm{~g}$ & $40 \mathrm{~g}$ & $38 \mathrm{~g}$ & $40 \mathrm{~g}$ \\
\hline Farinha de cascas de uvas & $1 \mathrm{~g}$ & - & $1 \mathrm{~g}$ & - & $1 \mathrm{~g}$ & - & $1 \mathrm{~g}$ & - \\
\hline Farinha de sementes de uvas & $1 \mathrm{~g}$ & - & $1 \mathrm{~g}$ & - & $1 \mathrm{~g}$ & - & $1 \mathrm{~g}$ & - \\
\hline Açúcar demerara & $19 \mathrm{~g}$ & $19 \mathrm{~g}$ & $19 \mathrm{~g}$ & $19 \mathrm{~g}$ & $19 \mathrm{~g}$ & $19 \mathrm{~g}$ & $19 \mathrm{~g}$ & $19 \mathrm{~g}$ \\
\hline Fermento químico em pó & $5 \mathrm{~g}$ & $5 \mathrm{~g}$ & $5 \mathrm{~g}$ & $5 \mathrm{~g}$ & $5 \mathrm{~g}$ & $5 \mathrm{~g}$ & $5 \mathrm{~g}$ & $5 \mathrm{~g}$ \\
\hline Ácido ascórbico & - & - & - & - & $3 \mathrm{~g}$ & $3 \mathrm{~g}$ & $2,5 \mathrm{~g}$ & $2,5 \mathrm{~g}$ \\
\hline Ácido cítrico & - & - & $1 \mathrm{~g}$ & $1 \mathrm{~g}$ & - & - & $0,5 \mathrm{~g}$ & $0,5 \mathrm{~g}$ \\
\hline Água potável & $60 \mathrm{~mL}$ & $60 \mathrm{~mL}$ & $60 \mathrm{~mL}$ & $60 \mathrm{~mL}$ & $60 \mathrm{~mL}$ & $60 \mathrm{~mL}$ & $60 \mathrm{~mL}$ & $60 \mathrm{~mL}$ \\
\hline Óleo de soja & $7 \mathrm{~mL}$ & $7 \mathrm{~mL}$ & $7 \mathrm{~mL}$ & $7 \mathrm{~mL}$ & $7 \mathrm{~mL}$ & $7 \mathrm{~mL}$ & $7 \mathrm{~mL}$ & $7 \mathrm{~mL}$ \\
\hline
\end{tabular}

$\mathrm{A} 1=$ Amostra 1; $\mathrm{C} 1=$ Controle 1; $\mathrm{A} 2=$ Amostra 2; $\mathrm{C} 2=$ Controle 2; $\mathrm{A} 3=$ Amostra 3; $\mathrm{C} 3=$ Controle 3; $\mathrm{A} 4=\mathrm{Amostra} 4 ; \mathrm{C} 4=\mathrm{Controle} 4$.

Foram utilizadas canecas de cerâmica, medindo $8,3 \mathrm{~cm}$ de altura e $7,5 \mathrm{~cm}$ de diâmetro, nas quais os bolos foram assados em forno micro-ondas (Panasonic Family, Brasil), potência $900 \mathrm{~W}$, por um período de 1 minuto e 30 segundos.

\subsection{Análises de polifenóis e atividade antioxidante}

A extração de polifenóis das amostras foi realizada conforme descrito por Larrauri et al. (1997). O conteúdo polifenólico total (CPT) foi determinado conforme Singleton \& Rossi (1965), sendo os resultados expressos em $\mathrm{mg}$ de ácido gálico equivalente por $100 \mathrm{~g}$ de base seca (mg GAE $100 \mathrm{~g}^{-1} \mathrm{bs}$ ). O método de diferença de $\mathrm{pH}$ foi utilizado para medir antocianinas monoméricas (AM) (Giusti \& Wrolstad, 2001) e os seus resultados foram expressos em mg de cianidina 3-glicosídeo por $100 \mathrm{~g}$ de base seca ( $\mathrm{mg} \mathrm{C} 3 \mathrm{G} 100 \mathrm{~g}^{-1} \mathrm{bs}$ ). Os flavonóis totais (FT) e os ácidos fenólicos (AF) foram determinados seguindo o procedimento descrito por Mazza et al. (1999) e os resultados foram expressos em $\mathrm{mg}$ de quercetina equivalente e $\mathrm{mg}$ de ácido cafeico equivalente por $100 \mathrm{~g}$ de base seca, respectivamente.

A análise antioxidante foi realizada pela determinação do ácido 2,2-azino-bis (3-etilbenzotiazolina-6-sulfônico) (ABTS) (Re et al., 1999) e do radical 2,2-difenil-1-picrilhidrazil (DPPH) (Brand-Williams et al., 1995). O Trolox foi utilizado como composto antioxidante padrão para ambos os testes e os resultados foram expressos em mmol de equivalente Trolox por $100 \mathrm{~g}$ de base seca (mmol TE $\left.100 \mathrm{~g}^{-1} \mathrm{bs}\right)$.

\subsection{Análise de cor}

Os parâmetros CIELab foram determinados usando iluminação difusa D-65 de um colorímetro Chroma Meter, Modelo CR-400, marca Konica Minolta. Os parâmetros medidos foram $L^{*}$ (luminosidade, abscissa de branco a preto), $a^{*}$ (coordenada vermelho/verde) e $b *$ (coordenada amarelo / azul).

\subsection{Análises de pH}

Para determinar o $\mathrm{pH}$ das amostras, foi seguido o método do Instituto Adolfo Lutz (2008) com poucas modificações, que foram: a água destilada para diluir a amostra foi fervida e resfriada, aproximadamente $1 \mathrm{~g}$ de amostra foi misturado com $10 \mathrm{~mL}$ desse diluente e, após $1 \mathrm{~h}$ no escuro, o material foi centrifugado (ITR, 
modelo: 8BT, Brasil). Em seguida, o sistema foi filtrado em papel Whatman $\mathrm{n}^{\circ} 1$ e o extrato aquoso foi submetido à análise de $\mathrm{pH}$.

\subsection{Volume específico e perda de massa}

O volume específico foi estabelecido pelo quociente entre o volume $\left(\mathrm{cm}^{3}\right)$ e a massa $(\mathrm{g})$ de cada amostra forneada, conforme Brito \& Cereda (2015), com resultados expressos $\mathrm{em} \mathrm{cm}^{3} \mathrm{~g}^{-1}$ e expressos em unidade de medida internacional $\left(\times 10^{-3} \mathrm{~m}^{3} \mathrm{~kg}^{-1}\right)$. A perda de massa foi expressa pela subtração do peso da massa úmida e peso do bolo assado. $\mathrm{O}$ volume foi obtido pela técnica de deslocamento de sementes de painço.

\subsection{Avaliação sensorial}

Foi realizado o teste afetivo de aceitação por escala hedônica (Instituto Adolfo Lutz, 2008), no qual 23 alunos e servidores, semitreinados e que já haviam realizado outras análises sensoriais de produtos com farinha de uva, indicando familiaridade com o produto, de uma Instituição de Ensino Superior do Rio Grande do Sul, expressaram os seus graus de gostar ou de desgostar dos bolos, de forma globalizada e em relação aos atributos aparência, cor, odor, maciez, granulometria, acidez, doçura, sabor, sabor residual e a aceitação global, dando uma nota de 1 a 7 a cada atributo de cada produto, sendo 1 correspondente a "desgostei muitíssimo", 4 "nem gostei nem desgostei" e 7 a "gostei muitíssimo". O estudo foi aprovado pelo Comitê de Ética em Pesquisa da Universidade Estadual do Rio Grande do Sul sob Parecer $n^{\circ} 3.466 .854$, CAAE: 15927219.2.0000.8091.

\subsection{Análises estatísticas}

As médias de triplicatas das amostras foram tratadas estatisticamente por Análise de Variância (ANOVA), seguidas por comparação pelo teste de Tukey por meio do Software Excel, sendo consideradas diferenças estatísticas quando $p<0,05$.

A correlação de Pearson foi realizada no software Excel ao comparar as médias dos experimentos, sendo considerada correlação forte, quando $r>0,7$; correlação moderada, quando $0,3<r<0,7$, e correlação fraca, quando $r<0,3$ (Figueiredo Filho \& Silva Júnior, 2009). Os resultados de análise sensorial foram tratados segundo a Análise Multivariada de Variância (MANOVA), utilizando o índice de Gower, e as medianas foram representadas nos gráficos de Boxplot bivariada no Software R, sendo consideradas diferenças estatísticas quando $p<0,05$.

\section{Resultados e discussão}

Os resultados da extração de polifenóis e compostos com atividade antioxidante nos bolos preparados encontram-se nas Tabelas 2 e 3, respectivamente.

De acordo com a Tabela 2, os bolos com farinha de cascas e sementes de uvas apresentaram quantidades de compostos fenólicos totais extraíveis semelhantes aos seus controles, o que pode indicar que a quantidade do subproduto não foi suficiente para aumentar os fenólicos no produto final ou que alguns fenólicos nas formulações com farinha de uva ficaram abaixo do limite detectável no método adotado. De acordo Sant'Anna et al. (2012), no bagaço de uva, existem grandes quantidades de polifenóis com atividade antioxidante que estão fortemente ligados à matriz vegetal, sendo extraíveis apenas em condições de alta força iônica. Observa-se, ainda, que os bolos com ácido ascórbico apresentaram teores de fenólicos totais superiores $(p<0,05)$ às amostras sem ácidos, sugerindo a interferência positiva do ácido ascórbico na estabilização e maior extração desses compostos. Em relação aos flavonóis totais, houve aumento significativo $(p<0,05)$ na amostra com farinha de uva e ácido cítrico (Amostra 2) em relação ao seu controle. 
Tabela 2. Resultados das quantidades de polifenóis e capacidade de atividade antioxidante dos bolos forneados.

\begin{tabular}{|c|c|c|c|c|}
\hline Bolos & $\begin{array}{c}\text { CPT } \\
\left(\mathrm{mg} \mathrm{GAE} 100 \mathrm{~g}^{-1} \mathrm{bs}\right)\end{array}$ & $\begin{array}{c}\mathrm{AF} \\
\left(\mathrm{mg} \mathrm{CAE} 100 \mathrm{~g}^{-1} \mathrm{bs}\right)\end{array}$ & $\begin{array}{c}\text { FT } \\
\left(\mathrm{mg} \mathrm{QE} 100 \mathrm{~g}^{-1} \mathrm{bs}\right)\end{array}$ & $\begin{array}{c}\mathrm{AM} \\
\left(\mathrm{mg} \mathrm{C3G} 100 \mathrm{~g}^{-1} \mathrm{bs}\right)\end{array}$ \\
\hline Amostra 1 & $1526,430 \pm 0^{c}$ & $8,680 \pm 0^{\mathrm{a}}$ & $53,055 \pm 3,458^{\mathrm{a}}$ & n.d. \\
\hline Controle 1 & $1392,525 \pm 11,505^{\mathrm{c}}$ & n.d. & n.d. & $0,673 \pm 0,952^{\mathrm{a}}$ \\
\hline Amostra 2 & $1577,670 \pm 22,076^{\mathrm{c}}$ & n.d. & $48,165 \pm 3,458^{\mathrm{ab}}$ & n.d. \\
\hline Controle 2 & $1418,620 \pm 8,697^{\mathrm{c}}$ & n.d. & $3,820 \pm 0^{c}$ & n.d. \\
\hline Amostra 3 & $2951,615 \pm 3,302^{a}$ & n.d. & $31,250 \pm 1,810^{\mathrm{ab}}$ & $8,501 \pm 5,465^{\mathrm{a}}$ \\
\hline Controle 3 & $2805,120 \pm 6,279^{a}$ & n.d. & n.d. & n.d. \\
\hline Amostra 4 & $2572,555 \pm 52,347^{b}$ & $2,240 \pm 3,168^{\mathrm{a}}$ & $25,545 \pm 10,133^{b c}$ & $12,245 \pm 9,168^{\mathrm{a}}$ \\
\hline Controle 4 & $2578,645 \pm 109,877^{b}$ & n.d. & n.d. & n.d. \\
\hline
\end{tabular}

Letras diferentes na mesma coluna significam que há diferenças estatísticas $(p<0,05)$. Médias \pm desvio padrão. Conteúdo fenólico total (CPT), ácidos fenólicos (AF), flavonóis totais (FT), antocianinas monoméricas (AM) e atividade antioxidante (ABTS e DPPH) dos bolos. n.d.: não detectado por estar abaixo do limite de quantificação.

Ao comparar as diferentes formulações com adição de farinha de uva, observou-se (Tabela 2) que aquelas com adição de ácido ascórbico ou combinação de ácido ascórbico e ácido cítrico aumenta $(p<0,05)$ a concentração de polifenóis totais extraíveis dos produtos assados. A adição de ácido cítrico de forma individual não afetou $(p>0,05)$ a concentração desses compostos. Em relação aos ácidos fenólicos, não houve diferença estatística $(p>0,05)$ entre as amostras adicionadas de farinha de uva, sem ácidos (Amostra 1) e com os dois ácidos (Amostra 4), não sendo detectados esses compostos nos demais tratamentos por estarem abaixo do limite de quantificação. Ao analisar a concentração de flavonóis totais das diferentes formulações com farinha de uva, os resultados mostram que a amostra sem ácidos apresentou o maior valor $(p<0,05)$ para esses compostos, em relação à amostra com os dois ácidos, não havendo diferença $(p>0,05)$ entre a primeira e as amostras com ácido cítrico ou com ácido ascórbico. Em relação às antocianinas monoméricas extraíveis, não houve diferença estatística $(p>0,05)$ entre as amostras que apresentaram valores dentro dos limites de deteç̧ão. Além das possibilidades de existência de antocianinas não extraíveis e não aciladas, este fato pode ser explicado por elas serem compostos fenólicos, instáveis e facilmente suscetíveis à degradação, podendo ser afetadas pelo $\mathrm{pH}$, temperatura, presença de enzimas, luz, oxigênio, estrutura e concentração das antocianinas, e presença de outros compostos, como outros flavonoides, proteínas e minerais (Rein, 2005; Liu et al., 2019).

De acordo com Amic et al. (1990) apud Žilić et al. (2016), em pH 5 e 6, antocianinas não aciladas são instáveis e descoloram rapidamente por hidratação na posição 2 da estrutura da antocianidina, formando as estruturas pseudobase de carbinol e chalcona. As antocianinas aciladas mostram uma melhor resistência a fatores, como calor (Delgado-Vargas et al., 2000). Como os pigmentos de antocianina são compostos lábeis (Žilić et al., 2016), sua cor pode ser estabilizada por copigmentação que, geralmente, envolve a formação de um complexo molecular não covalente com um composto orgânico ou não orgânico incolores. Essa estratégia pode ser usada para estabilizar as cores de antocianinas (Rein, 2005; Liu et al., 2019).

Em relação à extração de compostos com capacidade de sequestro de radicais ABTS e DPPH, verificouse (Tabela 3) que a adição de farinha de uva não acarretou aumento $(p>0,05)$ de atividade antioxidante. Já entre as formulações com farinha de uva, a amostra com adição de ácido ascórbico apresentou maior $(p<0,05)$ atividade antioxidante, medida pelo método ABTS, do que a amostra sem adição de ácidos. Entretanto, a formulação não apresentou diferença significativa $(p>0,05)$ quando comparada com a adição de ácido cítrico ou a combinação de ácidos. 
Efeito dos ácidos ascórbico e cítrico em propriedades físico-químicas e sensoriais de bolos vegetarianos com suplementação de farinha de bagaço de uva

Carlini, N. R. B. S. et al.

Tabela 3. Resultados da capacidade de atividade antioxidante dos bolos forneados.

\begin{tabular}{|c|c|c|}
\hline Bolos & ABTS activity (mmol TE $\left.100 \mathrm{~g}^{-1} \mathrm{bs}\right)$ & DPPH activity (mmol TE $100 \mathrm{~g}^{-1} \mathrm{bs}$ ) \\
\hline Amostra 1 & $36,405 \pm 5,021^{b}$ & $64,981 \pm 4,775$ bc \\
\hline Controle 1 & $42,971 \pm 8,070$ ab & $71,527 \pm 4,433 \mathrm{abc}$ \\
\hline Amostra 2 & $48,945 \pm 7,381 \mathrm{ab}$ & $57,378 \pm 5,127^{\mathrm{c}}$ \\
\hline Controle 2 & $47,200 \pm 3,466^{a b}$ & $75,326 \pm 5,513 a b c$ \\
\hline Amostra 3 & $59,638 \pm 1,841^{\text {a }}$ & $78,548 \pm 5,974$ abc \\
\hline Controle 3 & $62,875 \pm 0,851^{\text {a }}$ & $88,048 \pm 6,429$ ab \\
\hline Amostra 4 & $47,239 \pm 0,199 a b$ & $87,462 \pm 7,167$ ab \\
\hline Controle 4 & $43,924 \pm 4,019^{a b}$ & $94,620 \pm 10,206^{\mathrm{a}}$ \\
\hline
\end{tabular}

Letras diferentes na mesma coluna significam que há diferenças estatísticas $(p<0,05)$. Médias \pm desvio padrão. mmol TE $100 \mathrm{~g}^{-1}$ bs (mmol de equivalente Trolox por $100 \mathrm{~g}$ de base seca).

A análise de cor e a aparência dos produtos elaborados são evidenciadas na Tabela 4 e na Figura 1, respectivamente. Os resultados mostram que os bolos adicionados de farinha de uva apresentaram coloração mais escura $(p<0,05)$ quando comparados aos seus controles, comprovado pelo aumento do valor de $L^{*}$ nas amostras controles. Bender et al. (2016a) também verificaram que a inclusão de farinha de cascas de uvas diminuiu os valores de luminosidade das migalhas e crostas de muffins. Os resultados da análise das amostras adicionadas de farinha de uva, em relação ao parâmetro $a^{*}$, os resultados mostram que a adição de bagaço de uva na formulação sem ácidos acarretou uma coloração verde. Já o croma $b^{*}$ indicou uma tendência para a tonalidade azul nas amostras com o subproduto de uvas, variando entre 10,14 e 16,57. Resultados semelhantes foram observados no trabalho de Ortega-Heras et al. (2019), que analisaram muffins adicionados de pó de cascas de uvas.

Tabela 4. Cor da superfície dos bolos $\left(L^{*}, a^{*}, b^{*}\right)$.

\begin{tabular}{cccc}
\hline Formulações & $\boldsymbol{L}^{*}$ & $\boldsymbol{a}^{*}$ & $\boldsymbol{b}^{*}$ \\
\hline Amostra 1 & $44,42 \pm 0,14^{\mathrm{d}}$ & $-1,57 \pm 0,21^{\mathrm{d}}$ & $11,30 \pm 0,25^{\mathrm{c}}$ \\
\hline Controle 1 & $74,42 \pm 0,79^{\mathrm{ab}}$ & $-1,79 \pm 0,02^{\mathrm{d}}$ & $23,69 \pm 1,22^{\mathrm{a}}$ \\
\hline Amostra 2 & $52,63 \pm 0,14^{\mathrm{c}}$ & $5,09 \pm 0,04^{\mathrm{c}}$ & $10,14 \pm 0,15^{\mathrm{c}}$ \\
\hline Controle 2 & $77,26 \pm 2,11^{\mathrm{a}}$ & $-1,45 \pm 0,02^{\mathrm{d}}$ & $16,24 \pm 0,23^{\mathrm{b}}$ \\
\hline Amostra 3 & $49,28 \pm 0,57^{\mathrm{c}}$ & $7,33 \pm 0,03^{\mathrm{b}}$ & $10,91 \pm 0,01^{\mathrm{c}}$ \\
\hline Controle 3 & $73,77 \pm 1,10^{\mathrm{ab}}$ & $-1,7 \pm 0,07^{\mathrm{d}}$ & $16,87 \pm 0,05^{\mathrm{b}}$ \\
\hline Amostra 4 & $48,64 \pm 1,30^{\mathrm{cd}}$ & $7,87 \pm 0,23^{\mathrm{a}}$ & $10,24 \pm 0,78^{\mathrm{c}}$ \\
\hline Controle 4 & $71,50 \pm 0,38^{\mathrm{b}}$ & $-1,68 \pm 0,03^{\mathrm{d}}$ & $16,68 \pm 0,54^{\mathrm{b}}$ \\
\hline
\end{tabular}

$\overline{a, b, c, d}$ Diferentes letras sobrescritas na mesma coluna indicam diferença estatística $(p<0,05)$. Médias \pm desvio padrão.

Das formulações com farinha de uva, a amostra 1 foi a que apresentou o menor valor da coordenada de cor $a^{*}$. Esse resultado pode ser visível na Figura 1, em que claramente o bolo A1 apresenta cor esverdeada. A adição de ácidos minimizou esse efeito. A adição de ácido ascórbico ou sua combinação com ácido cítrico acarretou aumento $(p<0,05)$ do parâmetro $a^{*}$ (Tabela 4) e, consequentemente, ocorreu um deslocamento para o componente vermelho nos bolos (Figura 1). Na correlação de Pearson (Tabela 5), houve relação forte positiva entre $a^{*} \mathrm{e}$ antocianinas monoméricas, sugerindo maior estabilidade destes fenólicos nas formulações com os aditivos. 


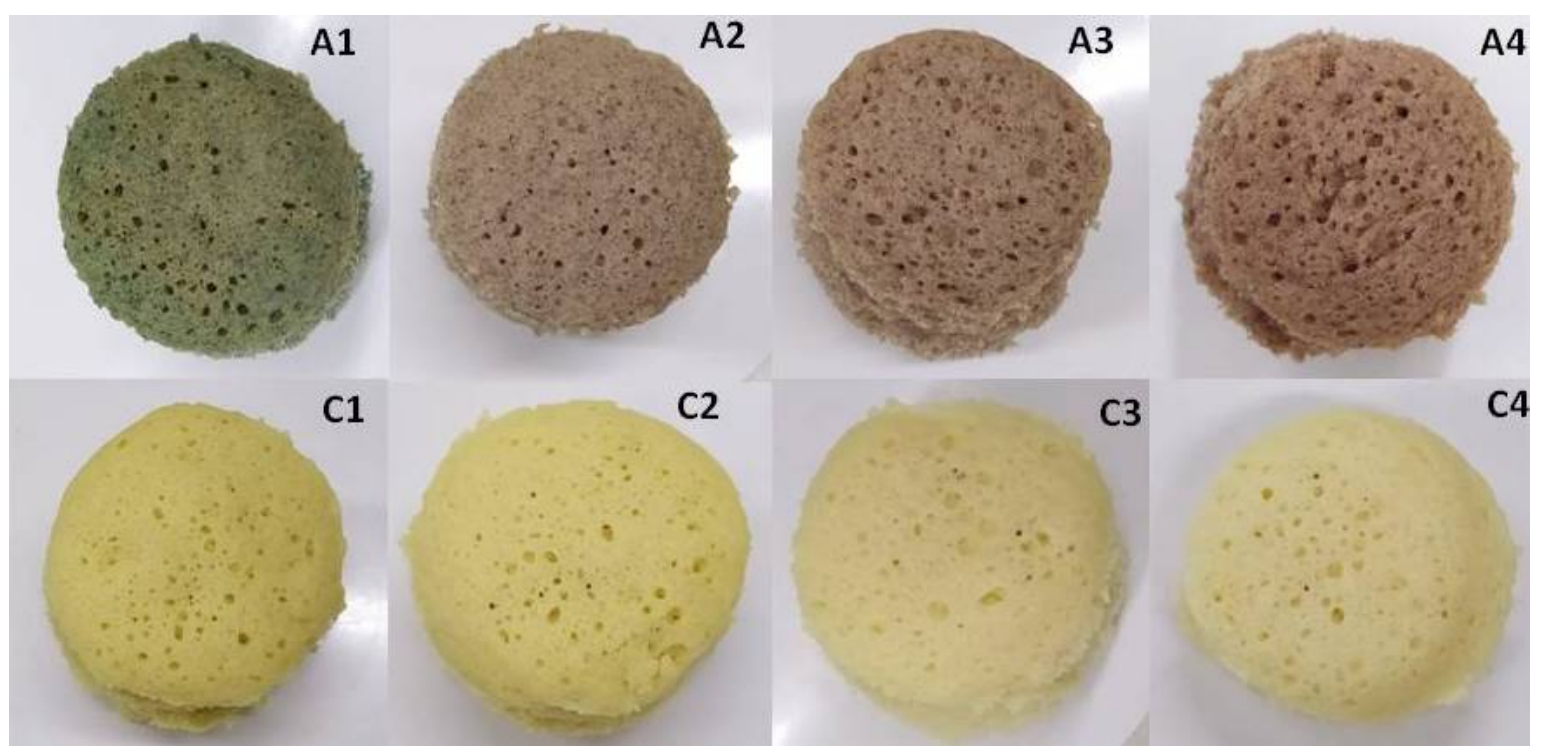

Figura 1. Fotos dos bolos desenvolvidos após o forneamento. Al= sem ácidos e com farinhas de cascas e sementes de uvas; $\mathrm{C} 1=$ sem ácidos e sem farinhas de cascas e sementes de uvas; $\mathrm{A} 2=$ com ácido cítrico e com farinhas de cascas e sementes de uvas; $\mathrm{C} 2=$ com ácido cítrico e sem farinhas de cascas e sementes de uvas; $\mathrm{A} 3=$ com ácido ascórbico e com farinhas de cascas e sementes de uvas; $\mathrm{C} 3=$ com ácido ascórbico e sem farinhas de cascas e sementes de uvas; A4= com ácido cítrico e ácido ascórbico e com farinhas de cascas e sementes de uvas; C4= com ácido cítrico e ácido ascórbico e sem farinhas de cascas e sementes de uvas.

Ao analisar o $\mathrm{pH}$ dos bolos, constatou-se que as amostras sem a adição de ácidos apresentaram valores de $\mathrm{pH}$ alcalinos $(8,82 \pm 0,06$ e $8,78 \pm 0,16)$, maiores $(p<0,05)$ do que aquelas com adição, que apresentaram valores de pH neutro (C2) a levemente ácidos (7,03 $\pm 0,01$ a 5,30 $\pm 0,05)$. A adição de ácido cítrico acarretou valores de $\mathrm{pH}$ maiores $(p<0,05)$ do que aqueles das amostras com ácido ascórbico ou combinação de ácidos.

Os resultados exibidos na Tabela 5 apresentam relação forte entre o $\mathrm{pH}$ dos bolos e os polifenóis extraíveis, e moderada ao ser relacionado à capacidade de sequestro de radicais ABTS e DPPH. Na correlação entre os polifenóis totais e a capacidade de sequestro de radicais DPPH e ABTS, percebe-se que a relação é de moderada a forte. Em relação à variável, antocianinas monoméricas, houve uma relação forte ao ser correlacionada ao parâmetro de cor $a^{*}$ e relação moderada quando comparada ao $\mathrm{pH}$. Assim, os resultados expostos na Tabela 5 indicam que quanto mais ácido (menor $\mathrm{pH}$ ) se mostrou o bolo, maior foi a tendência de aumento na concentração de polifenóis totais, antocianinas e compostos com atividade antioxidante, pelos métodos ABTS e DPPH.

Tabela 5. Valores do coeficiente de correlação de Pearson (r) entre os fenólicos, pH, parâmetro de cor $a^{*}$ e compostos com atividade antioxidante (ABTS e DPPH) analisados.

\begin{tabular}{ccccccccc}
\hline & CFT & FT & AF & AM & ABTS & DPPH & pH & $\boldsymbol{a}^{*}$ \\
\hline CFT & - & $-0,19$ & $-0,27$ & 0,54 & 0,68 & 0,75 & $-0,83$ & 0,40 \\
\hline FT & - & 0,62 & 0,19 & $-0,24$ & $-0,67$ & 0,16 & 0,49 \\
\hline AF & & - & $-0,02$ & $-0,59$ & $-0,31$ & 0,51 & $-0,13$ \\
\hline AM & & - & 0,24 & 0,31 & $-0,50$ & 0,83 \\
\hline ABTS & & & - & 0,33 & $-0,68$ & 0,32 \\
\hline DPPH & & & & - & $-0,62$ & $-0,07$ \\
\hline pH & & & & & - & $-0,54$ \\
\hline$a^{*}$ & & & & & & - & - \\
\hline
\end{tabular}

Conteúdo fenólico total (CFT), flavonóis totais (FT), ácidos fenólicos (AF), antocianinas monoméricas (AM), parâmetro de cor $\left(a^{*}\right) \mathrm{e}$ capacidade de sequestro de radicais ABTS e DPPH. 
Os dados da Tabela 5 corroboram com a discussão da relação da extração e estabilidade de polifenóis com a cor dos alimentos e seu pH. A coloração verde da amostra 1 (Figura 1) ocorreu, possivelmente, devido ao $\mathrm{pH}$ da formulação, sendo possível perceber que os bolos sem ácidos apresentaram $\mathrm{pH}$ entre 8,82 e 8,78 . As amostras 2 e 4 apresentaram valores de $\mathrm{pH}$ menores que os seus controles, considerando que o valor do $\mathrm{pH}$ das farinhas de casca e semente de uva encontrado neste estudo foi 3,76 $\pm 0,00$, o que está de acordo com o encontrado por Bender et al. (2016b) e Ferreira (2010), os quais obtiveram valores de $\mathrm{pH}$ em torno de 3,50 em farinhas de bagaço de uva, sendo classificadas como um produto muito ácido. Note-se que isto pode ter contribuído para os valores de $\mathrm{pH}$ dos bolos. $\mathrm{Na}$ análise das amostras com farinha de uva, as que continham ácido ascórbico em sua composição apresentaram os menores valores de $\mathrm{pH}(5,30 \pm 0,05$ e 5,48 $\pm 0,17)$. Em formulações ácidas, as antocianinas são vermelhas, mas, com o aumento do $\mathrm{pH}$, ocorre uma diminuição da intensidade de cor e de sua estabilidade química (Giusti \& Wrolstad, 2001).

Resultados semelhantes foram relatados por Žilić et al. (2016), quando farinhas de milho vermelhas e azuis foram usadas para a preparação de biscoitos funcionais, sendo observado que houve aumento significativo de compostos fenólicos nos biscoitos preparados a partir de milho azul, milho padrão azul e ácido cítrico. De acordo com os autores, o ácido cítrico afetou as antocianinas totais na massa de biscoitos de milho e nos biscoitos finais, diminuindo seu pH no sistema de massa e, por acilação, de seus resíduos de açúcar ou cátion flavílio. As cores visíveis observadas nas superfícies dos biscoitos com ácido cítrico foram rosa e, sem ácido cítrico, foram castanha e/ou verde.

Em relação ao percentual de perda de peso dos bolos, não houve diferença estatística $(p>0,05)$ entre as formulações, variando entre $8,98 \%$ e 11,33\% (Tabela 6). Já na análise de volume específico, com exceção do controle 4, no qual havia os dois ácidos, foi possível perceber que as formulações controles apresentaram os maiores volumes $(p<0,05)$ em relação às suas respectivas amostras com farinha de uva. Isso provavelmente ocorreu devido à substituição parcial da farinha de trigo por farinha de bagaço de uva, que causou a redução do glúten, diminuindo seus elementos estruturantes. Além disso, a fibra do subproduto de uva retém mais água durante a cocção, diminuindo a hidratação do glúten, o que pode ter contribuído na redução do volume (Samohvalova et al., 2016). A elevada absorção de água da farinha de bagaço de uva está associada, principalmente, ao seu grande teor de fibras (Porte et al., 2011; Santana et al., 2017).

Tabela 6. Perda de massa após forneamento e volume específico.

\begin{tabular}{ccc}
\hline Bolos & Perda de peso ao assar (\%) & Volume específico $\left(\times \mathbf{1 0}^{-\mathbf{3}} \mathbf{~ m}^{\mathbf{3}} \mathbf{k g}^{-\mathbf{1}}\right)$ \\
\hline Amostra 1 & $11,33 \pm 0,55^{\mathrm{a}}$ & $1,74 \pm 0,01^{\mathrm{b}}$ \\
\hline Controle 1 & $9,77 \pm 0,55^{\mathrm{a}}$ & $2,06 \pm 0,06^{\mathrm{a}}$ \\
\hline Amostra 2 & $11,33 \pm 0,55^{\mathrm{a}}$ & $1,71 \pm 0,06^{\mathrm{bd}}$ \\
\hline Controle 2 & $8,98 \pm 0,55^{\mathrm{a}}$ & $2,17 \pm 0,03^{\mathrm{a}}$ \\
\hline Amostra 3 & $10,55 \pm 1,66^{\mathrm{a}}$ & $1,36 \pm 0,16^{\mathrm{e}}$ \\
\hline Controle 3 & $10,16 \pm 0,00^{\mathrm{a}}$ & $1,69 \pm 0,06^{\mathrm{bcd}}$ \\
\hline Amostra 4 & $11,33 \pm 0,55^{\mathrm{a}}$ & $1,51 \pm 0,13^{\mathrm{de}}$ \\
\hline Controle 4 & $9,38 \pm 0,00^{\mathrm{a}}$ & $1,83 \pm 0,04^{\mathrm{abcd}}$ \\
\hline
\end{tabular}

$\overline{a, b, c, d, e}$ Diferentes letras sobrescritas na mesma coluna significam que há diferenças estatísticas $(p<0,05)$. Médias \pm desvio padrão.

A avaliação de diferentes formulações na aceitação de várias características sensoriais por meio da abordagem convencional "um atributo por vez" e da avaliação estatística convencional pode não levar a uma análise crítica e holística, ignorando a importância da interação dos parâmetros avaliados. A análise multivariada identifica interfaces semelhantes dos dados experimentais em que muitas características são 
reduzidas a variáveis inferiores, facilitando sua interpretação. Os resultados da análise sensorial são mostrados na Figura 2W, em que todos os parâmetros foram analisados juntamente e não atributo a atributo, como comumente é realizado. Essa estratégia permite que as aceitações das amostras sejam avaliadas em suas totalidades, considerando a análise conjunta dos diferentes atributos. Os resultados indicaram que as amostras com maiores aceitações, considerando todos os atributos avaliados, foram aquelas com farinha de uva e com ácido cítrico (C) e sem farinha de uva e com ácido cítrico (D), com farinha de uva e com ácido ascórbico (E) e sem farinha de uva e com ácidos cítrico e ascórbico (H). A maior mediana observada foi para o tratamento D (5), seguido de E (4,9), H e C (4,7), ainda que globalmente o maior valor seja atribuído ao tratamento $\mathrm{D}$, o qual é similar $(p>0,05)$ em aceitação para os tratamentos $\mathrm{E}$ e $\mathrm{H}$. Não houve diferença estatística $(p>0,05)$ entre a amostra A e a amostra E. No entanto, podemos verificar que individualmente os atributos possam variar entre os tratamentos (Figura 2Z). Com relação à análise isolada do atributo aparência, a amostra A obteve a menor mediana $(p<0,05)$ em relação à amostra E. Isto pode ser justificado pelo fato de o provador esperar que os produtos tenham uma cor característica, rejeitando produtos cuja cor não esteja de acordo com o esperado (Cobucci, 2010 apud Lermen et al., 2015), podendo a coloração verde da Amostra 1 (A) ter contribuído negativamente na sua avaliação.
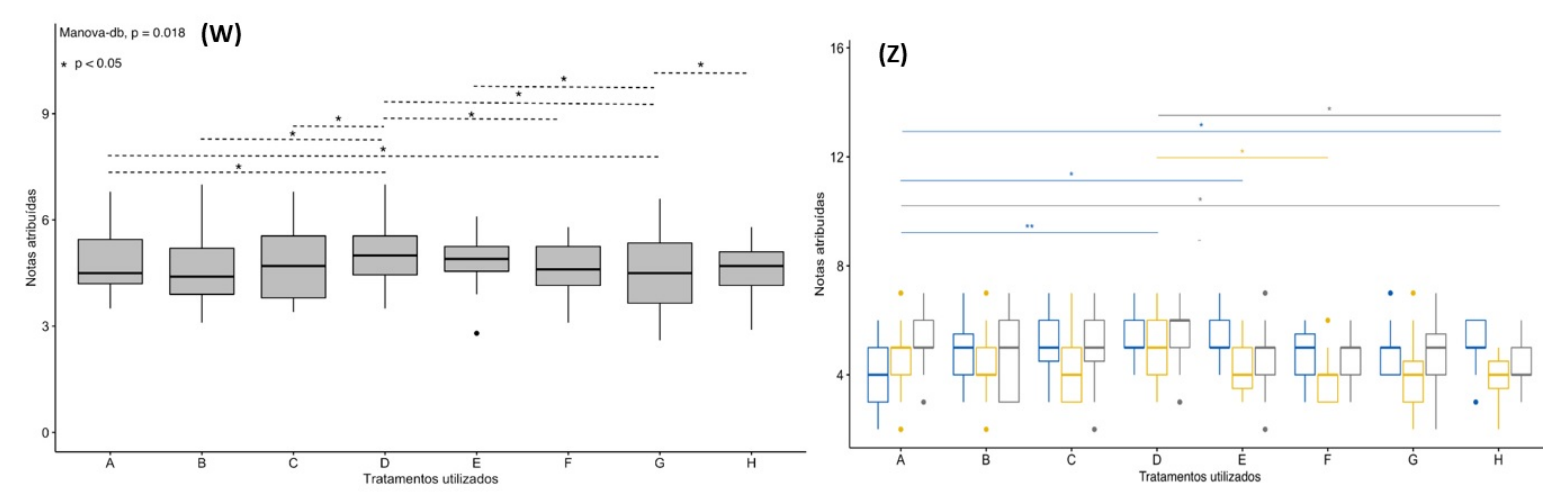

Figura 2. Análise do índice de Gower (W) e Análise de atributos sensoriais individuais (Z) para os atributos sensoriais dos bolos com substituição parcial de farinha de trigo por farinha de bagaço de uvas, com ou sem aditivos e seus respectivos controles. Somente pares significativos são mostrados. Com farinha de uva e sem ácidos (A), sem farinha de uva e sem ácidos (B), com farinha de uva e com ácido cítrico (C), sem farinha de uva e com ácido cítrico (D), com farinha de uva e com ácido ascórbico (E), sem farinha de uva e com ácido ascórbico (F), com farinha de uva e com ácidos cítrico e ascórbico $(\mathrm{G})$ e sem farinha de uva e com ácidos cítrico e ascórbico $(\mathrm{H})$. Figura 2Z: Aparência (azul), odor (amarelo) e maciez (cinza).

Alguns estudos têm verificado que a adição de farinha de bagaço de uva em produtos panificados, como muffins (Bender et al., 2016a), cookies (Poiani \& Montanuci, 2019), biscoitos amanteigados (Samohvalova et al., 2016), pães e brownies (Walker et al., 2014) tem provocado boa aceitação global nos provadores, demonstrando o potencial de mercado para esses produtos alimentícios.

\section{Conclusões}

A incorporação dos ácidos ascórbico e cítrico nos bolos com farinha de bagaço de uva possivelmente acarretou estabilização da coloração vermelha, de acordo com o método CIELab. Além disso, as formulações com ácido ascórbico apresentaram maiores teores de fenólicos totais e atividade antioxidante em relação às amostras sem ácidos, sugerindo a interferência positiva do ácido ascórbico na estabilização e maior extração desses compostos.

O bolo com farinha de bagaço de uva e ácido ascórbico apresentou as maiores quantidades de compostos fenólicos totais quando comparado aos outros bolos com o subproduto, além de ter sido um dos bolos mais bem aceitos pelos provadores, demonstrando o potencial de mercado para esses produtos alimentícios 
inovadores. Dessa forma, esta destinação do subproduto do processamento da uva é uma alternativa interessante que pode contribuir para o gerenciamento de resíduos industriais.

\section{Agradecimentos}

Os autores agradecem à Universidade Estadual do Rio Grande do Sul (UERGS) e ao Instituto Federal de Educação, Ciência e Tecnologia do Sertão Pernambucano (IF Sertão-PE) pelo financiamento. Os autores agradecem à EcoNatura pela doação das farinhas de cascas e sementes de uvas.

\section{Referências}

Bender, A. B. B., Luvielmo, M. D. E. M., Loureiro, B. B., Speroni, C. S., Boligon, A. A., Silva, L. P. D. E., \& Penna, N. G. (2016b). Obtenção e caracterização de farinha de casca de uva e sua utilização em snack extrusado. Brazilian Journal of Food Technology, 19, e2016010. http://dx.doi.org/10.1590/1981-6723.1016

Bender, A. B. B., Speroni, S. C., Salvador, P. R., Loureiro, B. B., Lovatto, N. M., Goulart, F. R., Lovatto, M. T., Miranda, M. Z., Silva, L. P., \& Penna, N. G. (2016a). Grape pomace skins and the effects of its inclusion in the technological properties of muffins. Journal of Culinary Science \& Technology, 15(2), 143-157. http://dx.doi.org/10.1080/15428052.2016.1225535

Brand-Williams, W., Cuvelier, M. E., \& Berset, C. (1995). Use of a free radical method to evaluate antioxidant activity. Lebensmittel-Wissenschaft + Technologie, 28(1), 25-30. http://dx.doi.org/10.1016/S0023-6438(95)80008-5

Brito, V. H. dos S., \& Cereda, M. P. (2015). Método para determinação de volume específico como padrão de qualidade do polvilho azedo e sucedâneos. Brazilian Journal of Food Technology, 18(1), 14-22. http://dx.doi.org/10.1590/1981-6723.0214

Cabral, C. B., Quadros, C. P., \& Silva, C. S. (2020). Sweet type brigadeiro made with banana biomass and flour grape residue from the wine production of the region of the submédio São Francisco. Brazilian Journal of Development, 6(6), 40654-40664. http://dx.doi.org/10.34117/bjdv6n6-559

Delgado-Vargas, F., Jiménez, A. R., \& Paredes-López, O. (2000). Natural pigments: Carotenoids, anthocyanins, and betalains: Characteristics, biosynthesis, processing, and stability. Critical Reviews in Food Science and Nutrition, 40(3), 173-289. PMid:10850526. http://dx.doi.org/10.1080/10408690091189257

Ferreira, L. F. D. (2010). Obtenção e caracterização de farinha de bagaço de uva e sua utilização em cereais matinais expandidos (Tese de doutorado). Universidade Federal de Viçosa, Viçosa.

Figueiredo Filho, D. B., \& Silva Júnior, J. A. (2009). Desvendando os mistérios do coeficiente de correlação de Pearson (r). Revista Política Hoje, 18(1), 115-146. Recuperado em 9 de outubro de 2020, de https://periodicos.ufpe.br/revistas/politicahoje/article/viewFile/3852/3156

Giusti, M., \& Wrolstad, R. E. (2001). Characterization and measurement of anthocyanins by UV-visible spectroscopy. Current Protocols in Food Analytical Chemistry, (1), 1-13. http://dx.doi.org/10.1002/0471142913.faf0102s00

Instituto Adolfo Lutz - IAL. (2008). Métodos físico-químicos para análise de alimentos (4. ed., 1018 p.). São Paulo: IAL.

Ishimoto, E. Y. (2008). Efeito hipolipemiante e antioxidante de subprodutos da uva em hamsters (Tese de doutorado). Universidade de São Paulo, São Paulo.

Larrauri, J. A., Rupérez, P., \& Saura-Calixto, F. (1997). Effect of drying temperature on the stability of polyphenols and antioxidant activity of red grape pomace Peels. Journal of Agricultural and Food Chemistry, 45(4), 1390-1393. http://dx.doi.org/10.1021/jf960282f

Lermen, F. H., Matias, G. de S., Modesto, F. A., Röder, R., \& Boikos, T. J. P. (2015). Teste de consumidores e análise de aparência, sabores e cores para o desenvolvimento de novos produtos: O case do projeto de broinhas de milho saboreadas. Revista Latino-America de Inovação e Engenharia de Produção, 3(4), 97-109. http://dx.doi.org/10.5380/relainep.v3i4.37744

Liu, J., Tan, Y., Zhou, H., Muriel Mundo, J. L., \& Mcclements, D. J. (2019). Protection of anthocyanin-rich extract from pHinduced color changes using water-in-oil-in-water emulsions. Journal of Food Engineering, 254, 1-9. http://dx.doi.org/10.1016/j.jfoodeng.2019.02.021

Mazza, G., Fukumoto, L., Delaquis, P., Girard, B., \& Ewert, B. (1999). Anthocyanins, phenolics, and color of cabernet franc, merlot, and pinot noir wines from British Columbia. Journal of Agricultural and Food Chemistry, 47(10), 4009-4017. PMid:10552758. http://dx.doi.org/10.1021/jf990449f

Monagas, M., Hernández-Ledesma, B., Gómez-Cordovés, C., \& Bartolomé, B. (2006). Chemical characterization of commercial dietary ingredients from vitis vinifera I. Analytica Chimica Acta, 563(1-2), 401-410. PMid:16417286.

http://dx.doi.org/10.1016/j.aca.2005.10.065

Monrad, J. K., Howard, L. R., King, J. W., Srinivas, K., \& Mauromoustakos, A. (2010). Subcritical solvent extraction of anthocyanin from dried red grape pomace. Journal of Agricultural and Food Chemistry, 58(5), 2862-2868. PMid:20148515. http://dx.doi.org/10.1021/jf904087n

Ortega-Heras, M., Gómez, I., Pablos-Alcalde, S., \& González-Sanjosé, M. L. (2019). Application of the just-about-right scales in the development of new healthy whole-wheat muffins by the addition of a product obtained from white and red grape pomace. Foods, 8(9), 419. PMid:31533219. http://dx.doi.org/10.3390/foods8090419 
Pereira, E. P. R., Amorim, E. O. C., Ambiel, H. C. I., Chang, Y. K., \& Steel, C. J. (2009). Influência de agentes oxidantes sobre as propriedades reológicas de massas de farinha de trigo branca e de grão inteiro e sobre o volume específico de pão francês. Brazilian Journal of Food Technology, 12(3), 161-171. http://dx.doi.org/10.4260/BJFT2009800900009

Poiani, M. R., \& Montanuci, F. D. (2019). Physical, technological characterization and texture profile of grapefruit and flaxseed cookies. Brazilian Journal of Food Technology, 22, e2018074. http://dx.doi.org/10.1590/1981-6723.07418

Porte, A., Silva, E. F., Almeida, V. D. S., Silva, T. X., \& Porte, L. H. M. (2011). Propriedades funcionais tecnológicas das farinhas de sementes de mamão (Carica papaya) e de abóbora (Cucurbita sp). Revista Brasileira de Produtos Agroindustriais, 13(1), 91-96. http://dx.doi.org/10.15871/1517-8595/rbpa.v13n1p91-96

Re, R., Pellegrini, N., Proteggente, A., Pannala, A., Yang, M., \& Rice-Evans, C. (1999). Antioxidant activity applying an improved ABTS radical cation decolorization assay. Free Radical Biology \& Medicine, 26(9-10), 1231-1237. PMid:10381194. http://dx.doi.org/10.1016/S0891-5849(98)00315-3

Rein, M. J. (2005). Copigmentation reactions and color stability of berry anthocyanins (Dissertation). University of Helsinki, Department of Applied Chemistry and Microbiology, Helsinki.

Révillion, J. P., Kapp, C., Badejo, M. S., \& Dias, V. V. (2020). O mercado de alimentos vegetarianos e veganos: Características e perspectivas. Cadernos de Ciência \& Tecnologia, 37(1), e26603. http://dx.doi.org/10.35977/0104-1096.cct2020.v37.26603

Samohvalova, O., Grevtseva, N., Brykova, T., \& Grigorenko, A. (2016). The effect of grape seed powder on the quality of butter biscuits. Eastern-European Journal of Enterprise Technologies, Kharkov, 3(11(81)), 61-66. http://dx.doi.org/10.15587/17294061.2016.69838

Sant'Anna, V., Brandelli, A., Marczak, L. D. F., \& Tessaro, I. C. (2012). Kinetic modeling of total polyphenol extraction from grape marc and characterization of the extracts. Separation and Purification Technology, 100, 82-87. http://dx.doi.org/10.1016/j.seppur.2012.09.004

Santana, F. C., Silva, J. V., Santos, A. J. A. O., Alves, A. R., Wartha, E. R. S. A., Marcellini, P. S., \& Silva, M. A. A. P. (2011). Desenvolvimento de biscoito rico em fi bras elaborado por substituição parcial da farinha de trigo, por farinha da casca do maracujá amarelo (Passifl ora edulis flavicarpa) e fécula de mandioca (Manihot esculenta crantz). Alimentos e Nutrição, 22(3), 391-399. Recuperado em 9 de outubro de 2020, de http://servbib.fcfar.unesp.br/seer/index.php/alimentos/article/viewArticle/1646

Santana, G. S., Oliveira Filho, J. G., \& Egea, M. B. (2017). Características tecnológicas de farinhas vegetais comerciais. Revista de Agricultura Neotropical, 4(2), 88-95.http://dx.doi.org/10.32404/rean.v4i2.1549.

Saura-Calixto, F. (1998). Antioxidant dietary fiber product: A new concept and a potencial food ingredient. Journal of Agricultural and Food Chemistry, 46(10), 4303-4306. http://dx.doi.org/10.1021/jf9803841

Singleton, V. L., \& Rossi, J. A. (1965). Colorimetry of total phenolics with phosphomolybdic-phosphotungstic acid reagents. American Journal of Enology and Viticulture, 20, 144-158. Recuperado em 9 de outubro de 2020, de https://www.ajevonline.org/content/16/3/144

Toaldo, I. M., Cruz, F. A., Alves, T. de L., de Gois, J. S., Borges, D. L. G., Cunha, H. P., da Silva, E. L., \& Bordignon-Luiz, M. T. (2015). Bioactive potential of Vitis labrusca L. grape juices from the Southern Region of Brazil: Phenolic and elemental composition and effect on lipid peroxidation in healthy subjects. Food Chemistry, 173, 527-535. PMid:25466055. http://dx.doi.org/10.1016/j.foodchem.2014.09.171

Tonon, R. V., Silva, C. M., Galdeano, M. C., \& dos Santos, K. M. O. (2018). Tecnologias para o aproveitamento integral dos resíduos da indústria vitivinícola. Rio de Janeiro: Embrapa Agroindústria de Alimentos. 41p.

Valiente, C., Arrigoni, E., Esteban, R. M., \& Amado, R. (1995). Grape pomace as a potential food fiber. Journal of Food Science, 60(4), 818-820. http://dx.doi.org/10.1111/j.1365-2621.1995.tb06237.x

Walker, R., Tseng, A., Cavender, G., Ross, A., \& Zhao, Y. (2014). Physicochemical, nutritional, and sensory qualities of wine grape pomace fortified baked goods. Journal of Food Science, 79(9), S1811-S1822. PMid:25102950. http://dx.doi.org/10.1111/1750-3841.12554

Žilić, S., Kocadağli, T., Vančetović, J., \& Gökmen, V. (2016). Effects of baking conditions and dough formulations on phenolic compound stability, antioxidant capacity and color of cookies made from anthocyanin-rich corn flour. Lebensmittel-Wissenschaft + Technologie, 65, 597-603. http://dx.doi.org/10.1016/j.Iwt.2015.08.057

Financiamento: Nenhum. 